\title{
MUDANZAS EN PORTUGAL, EN EL CONTEXTO DE LA PENÍNSULA IBÉRICA
}

\section{Lorenzo LÓPEZ TRIGAL}

Departamento de Geografía y Geología. Universidad de León

\section{PALABRAS SENTIDAS PREVIAS}

En el presente Homenaje al profesor Jorge Gaspar, los organizadores me han invitado para que interviniera acerca de los cambios ocurridos en Portugal desde la premisa de una mirada desde España, observatorio privilegiado en este caso, en cuanto que portugueses y españoles somos "territoriantes" de una misma unidad vecinal, el solar ibérico, y "descendientes" de un mismo pueblo originario, aunque tamizado por el devenir posterior, unas veces unidos y otras de espaldas, de ahí que permítanme que presuma que los españoles seamos aquí "menos de fuera" que el resto de europeos. Si bien, en este último tiempo, el desarrollo de la España de las Autonomías ha derivado en una mayor diversificación de la visión española, al mismo tiempo que se introduce un sesgo de "nuevas penínsulas" y de cierto distanciamiento de los habitantes del Este español, más proclives a conocer las ciudades del centro de Europa, que a los "finisterres" del resto del Oeste de España y, de paso, de Portugal.

Por mi parte, me siento más bien transfronterizo en el interés geográfico y viajero y, en cierta forma, atraído por "los vientos del Oeste que llevan hasta León el olor a pinos de Portugal", como escribiera mi paisano y poeta Antonio Pereira. En efecto, discurría el año 1970, cuando por primera vez me asomara a la Raya de Vilar Formoso, coincidiendo, sin saberlo, en un día de marcada identificación nacional por la visita del presidente Marcelo Caetano a esta localidad. Fue una visita muy corta y de la que guardo dos escenas retrospectivas: escolares con banderitas y mujeres portadoras transitando por la senda al pie del ferrocarril internacional lejos del paso fronterizo. Cuatro años después, al mes de la revolución de abril, volvía con mi familia en un viaje de descubrimiento del

\footnotetext{
${ }^{1}$ Nota previa: el texto corresponde a la intervención en el Seminario Internacional realizado en el ámbito de Homenaje a Jorge Gaspar, el 28 de noviembre de 2007, en el Salão Nobre, Reitoria da Universidade de Lisboa
} 
Norte de Portugal del que retornamos contagiados del fervor popular de aquellos días. Al año siguiente, y desde entonces de manera ininterrumpida, seguiría la visita a Lisboa con un primer encuentro con Jorge Gaspar y colegas lisboetas que será el preludio de una estrecha colaboración y amistad.

Por todo ello, en diferentes etapas de mi investigación en estos años he venido recalando en fenómenos como la emigración portuguesa, la articulación territorial en las regiones de la Raya y últimamente sobre las ciudades portuguesas, es decir, he espigado en algunas cuestiones que no en una obra de síntesis, como fuera la de nuestro amigo el finado François Guichard. Eso sí, confieso aquí una frustración particular, la de no haber llevado adelante una obra colectiva sobre "la ciudad, la metrópoli y la región en el ámbito ibérico", que pergeñara junto al profesor Gaspar hace algún tiempo.

Pues bien, ¿qué ha ocurrido en este tiempo en Portugal que me llame más la atención como español? A falta de una amplia oleada de encuestas que lo definiera de forma mucho más ajustada a la realidad, creo no equivocarme mucho si manifiesto que sorprende gratamente ciertos rasgos geográficos culturales observados en la sociedad portuguesa, esto es: su modo de vida, más apegado al hogar y al terreno; su identidad territorial, más nacional y local que regional; su apertura al mundo, con más ánimo de entenderse en otras lenguas y de escudriñar el horizonte. Desde estas tres cualificaciones preliminares, que me parecen esenciales en el modo de comprensión diferencial de la sociedad portuguesa en parangón con España, pasaré a referir algunas miradas dispares con un cierto hilo conductor, apoyándome en los escritores viajeros y geógrafos españoles, las interrelaciones socioeconómicas Portugal-España y la reconfiguración del sistema territorial y urbano ibérico.

\section{VIAJEROS Y GEÓGRAFOS ESPAÑOLES AL "PAÍS VECINO TRAS LA RAYA"}

En marzo de 1972, Eduardo Barrenechea y Antonio Pintado publicaban en el diario madrileño 'Informaciones' un excelente Viaje a la frontera del subdesarrollo que dará una llamada de atención sobre la situación común de "una gran bolsa de atraso, salpicada -casi únicamente en el lado español- por tres o cuatro oasis de una relativa pujanza", donde destacarán el centralismo imperante de ambas capitales, aunque más agudo en Portugal y donde, además, la penuria estadística es notable tanto por la escasez de la misma como por el atraso de su publicación. Los resultados de este viaje informativo, cuyo propósito -confiesan los autores- fue "contribuir a que comencemos a vernos las caras y encarar juntos problemas comunes", tendrán amplia repercusión en años siguientes tras su publicación como libro en sendas ediciones en las dos lenguas. Pasado el tiempo, creo que muchos de los estudiosos de las ciencias sociales, como tam- 
bién los políticos, hemos asumido tal propósito-proyecto y volcado ese mismo interés en nuestras investigaciones, así como ha servido de orientación a los programas pilotos de los años 1980 y las posteriores actuaciones de cooperación transfronteriza.

Hay que hablar aquí del cambio producido en esos mismos años en la frontera, que hasta entonces se caracterizaba por ser puerta de fortín, con parada doble obligatoria y documentación en mano, carta verde e impreso de estancia para entregar a la salida... cuando no se daba la desconfianza de "guardas" y "guardinhas", en su celo de inspección del interior del maletero del vehículo y preguntarnos ¿para qué llevan Ustedes tanto libro y papel? La mudanza ha sido bien notoria: en los últimos años será todo coser y cantar, sin paradas ni guardas, pues la Raya ha dejado de ser "frontera exterior nacional" para ser una "frontera interior comunitaria" de controles esporádicos a bastante distancia de la linde, que ha tenido aún mayor apertura con el uso de la moneda común. Ello facilita la labor y aminora, de paso, el estrés anterior del viajero por carretera, aunque pierda en aventura y un tanto del desconcierto habitual y la sorpresa que conlleva todo itinerario inicial transfronterizo, aunque restan aún los vestigios de aduanas y "alfândegas" con rotulaciones tan llamativas como "Portugal no es un país pequeño" junto al mapa europeo sobreimpuesto al colonial africano portugués, y todavía más al interior del país puede sorprendernos el paisaje urbano diferente de las chimeneas y tejados, las Casas de Misericordia, las fortalezas de piedra oscura y sus iglesias encastradas o las ciudadelas, como también la mayor abundancia de floresta en el Norte y el paisaje de los montados en el Sur del país lusitano... Todo nos puede parecer diferente a lo que dejamos atrás, si bien es perceptible que las diferencias son menos nítidas, en transición en todo caso, en algunos territorios como la región miñota, la menos dispar de la Raya.

Es así que, a la llamada de atención de una mayor interrelación ibérica nos sumaremos geógrafos de distintas universidades españolas, desde el inicio de los Coloquios Ibéricos de Geografía a partir de 1979 y animados por los catedráticos Ángel Cabo Alonso en Salamanca y Joan Vilá Valentí en Barcelona, autor éste de una conocida monografía en francés y español que destaca en su línea de investigación de análisis geográfico regional (La Península ibérica, 1968) con versión italiana y alemana, hecho excepcional en un colega español, y revisada y puesta al día en español en 1989 y en cuya presentación se planteaba si "¿la Península ibérica es puente intercontinental y encrucijada o mole casi inexpugnable y bastión defensivo?, pues por la posición del solar ibérico más bien tiende a lo primero, mas la configuración interna favorece lo segundo y dificulta la penetración, de ahí que el estudio de los hechos humanos muestra respuestas encontradas y paradójicas a lo largo de la historia". Sin embargo, el profesor Vilá no abordará la incidencia como tal del fenómeno de la frontera o 
las interrelaciones socioeconómicas y flujos entre ambos países, sino más bien la organización territorial y política en el contexto del nuevo proceso de integración europea, tal como van a ser algunas de las aproximaciones de geógrafos posteriores. Digamos que con Vilá se cierra la etapa anterior de estudios ibéricos de geografía regional y dará paso a una nueva etapa -aún inicial- con investigaciones de ciertos temas relevantes de tipo social y territorial, y con una visión encaminada a entender el iberismo desde la cooperación abierta y la integración europea, como señalara el profesor Valentín Cabero en el acto de apertura del curso académico en la Universidad de Salamanca en el año 2002.

En este camino, en paralelo a ciertos escritores como el leonés Julio Llamazares, con un recorrido denso por Trás-os-Montes en 1996, la respuesta de los geógrafos va a ser desigual (L. LóPEZ TRIGAL, «La Raya ibérica como espacio de atracción y cooperación», Papeles de Economía Española, Economía de las Comunidades Autónomas, 21, 2005, pp. 3-12), pero tiene la virtud de mostrar nuevas inquietudes e intereses en el ámbito de la Raya, pues esta frontera es "un estado de ánimo, como el fado o la saudade, que te invade y que ya no te abandona" en palabras sentidas de Llamazares. Las investigaciones fronterizas se han centrado en el efecto frontera en el territorio rayano y la sociedad local y regional del Portugal interior y del Oeste de España. Si bien es cierto que se han demorado otros estudios sectoriales o generales sobre Portugal, sus ciudades y territorios, entre los geógrafos españoles, más animados en estos años por la investigación de países latinoamericanos o, sobre todo, de sus propias regiones y lugares. Se echa en falta, en cualquier caso, equipos consolidados de investigación en este campo, a pesar de que exista una cierta infraestructura de apoyo desde la 'Fundación Rei Afonso Henriques' en Zamora y el 'Centro de Estudos Ibéricos' en Guarda.

\section{EL EMPRESARIADO "A LA CONQUISTA DEL MERCADO VECINO"}

Según el lusitanista Hipólito de la Torre "esa formidable frontera secante luso-española... fue sobre todo la consecuencia de una estrategia nacionalista que se rastrea sin dificultad hasta los últimos años... en que pese a residuales desconfianzas y complejos, pese a legítimos apuntalamientos políticos y culturales de las dos realidades nacionales... la frontera se torna crecientemente porosa, si bien es cierto que mucho más la que comunica a las naciones que a las regiones, donde el estigma histórico del despoblamiento y el subdesarrollo aún están lejos de ser un recuerdo del pasado" (V. MORALES LEZCANO, Las fronteras de la Península ibérica en los siglos XVIII y XIX, Madrid, UNED, 2000, pp. 13 y 16). Efectivamente, nos encontramos ahora con un periodo de mayor intensidad de las relaciones, sobre todo comerciales y económicas, como también culturales y 
sociales en las dos direcciones, a la vez que asistimos a una crisis que se establece, de un lado, entre la emergencia de la modernidad que presta el marco europeo y la pervivencia de la tradición, y de otro lado, entre el reencuentro y el recelo de unos y otros.

Las economías portuguesa y española han mudado de raíz a partir de la entrada en la Comunidad Europea hace ya veinte años y se constata una mayor apertura de las relaciones a nivel internacional e ibérico, con un incremento sustancial del comercio exterior de ambos países. A la vez, las dos economías ibéricas son favorecidas por el incremento de las relaciones que se desarrollan siempre con una diferencia a favor de España de al menos 2 a 3 veces de valor, como resultado de ser una economía más fuerte y algo más competitiva, que se traduce en una proporción de 1 a 5 en cuanto al valor añadido bruto, con lo que se puede advertir una posición no tan desfavorable para Portugal respecto de esta relación comercial, por lo que no es de extrañar que esta relación haya sido diagnosticada "de vecinos distantes a asociados inquietos", como muestran las precauciones habidas en el proceloso mundo de la inversión y la banca (W. CHISLETT, 2004, Spain and Portugal: from distant neighbours to uneasy associates, in: htpp://www.realinstitutoelcano.org/documentos/134.asp).

Esto ha supuesto, se mida como se mida, un extraordinario cambio cuantitativo, pero asimismo han cambiado los componentes del comercio exterior hispano-luso, ahora en un mercado interrelacionado entre empresas y filiales de un lado y otro, derivado de las estrategias de "iberización" de algunas entidades a un ritmo tendencial progresivo. De ahí que, en paralelo, se hayan multiplicado las inversiones directas españolas y portuguesas en los respectivos países, en especial las primeras, dirigidas a la banca, el comercio, energía, telecomunicación, construcción, transporte, turismo e industria diversa, en forma de participaciones cruzadas e intercambio de acciones en empresas de uno y otro lado, lo que está produciendo de hecho flujos de capitales continuos hacia las áreas metropolitanas y principales corredores interurbanos y nuevas formas de relacionarse y flujos de ejecutivos.

Mas, convengamos, que esta nueva situación se aleja de la tozuda realidad que rodea a los espacios interiores y periféricos, vocacionados sus puntos más accesibles a servir de de mercados fronterizos (Valença do Minho, Chaves, Miranda do Douro, Vilar-Formoso, Elvas), aunque atraviesan en la actualidad por una crisis de ventas y de merma de atracción de clientela española. Asimismo, la inversión directa portuguesa en España, en su distribución espacial, ignora hasta ahora los archipiélagos y ciertas provincias interiores y del litoral, concentrándose sobre todo en las de Madrid y Barcelona. Se percibe, además, que en ciertos sectores del mercado ibérico no se avanza a igual ritmo en la interrelación, como es el caso del mercado eléctrico, el acoplamiento del desarrollo de 
ferrocarril de alta velocidad o sobre todo el planeamiento territorial transfronterizo.

\section{CAMBIOS EN EL SISTEMA TERRITORIAL Y URBANO IBÉRI- CO}

Es preciso dejar constancia en este apartado, en primer término, la dinámica y el papel de las áreas metropolitanas y las áreas urbanas, de ciertos corredores y ejes interurbanos que colaboran a la reconfiguración o recomposición del sistema territorial ibérico. En este marco el esquema de ocupación del espacio portugués es el de una desigual urbanización y densidad de poblamiento, con ciertas tendencias dominantes en cuanto a la concentración urbana litoral, el mantenimiento de la bipolarización del sistema urbano (Lisboa-Porto), el aumento de los flujos y de las interdependencias territoriales como rasgo de la nueva sociedad-red, o el alargamiento de las cuencas de empleo a través de los movimientos pendulares residencia-trabajo, que llegan a convertirse en transfronterizos de ciclo diario o semanal en ciertas áreas del Norte de Portugal.

Pero cabe preguntarse si el refuerzo del bipolo metropolitano Lisboa-Porto ¿no conducirá a mantener la casi ausencia de ciudades medias? Esta es la tesitura en la que se encuentra el sistema urbano portugués y esto marca, evidentemente, a la hora de interrelacionar los dos sistemas nacionales urbanos, especialmente en las regiones fronterizas, donde las ciudades de la raya portuguesa se encuentran siempre por bajo de la talla de las ciudades rayanas españolas, y donde la relación poblacional y funcional entre ciudades vecinas de uno y otro lado de la frontera se encuentra normalmente desajustada por parte de Portugal. Los espacios del interior portugués son, si cabe, más periféricos y están más desprovistos de ciudades que sus vecinos españoles, caracterizándose por la presencia de pequeñas ciudades y núcleos semiurbanos poco atrayentes funcionalmente y estrechamente dependientes de las metrópolis a cuya atracción es difícil resistir.

Sin embargo, en los últimos años se aprecia una tendencia y esfuerzo en las políticas públicas en cuanto a un cierto crecimiento autónomo en algunas ciudades (Braga y su entorno) y ejes en ciernes (destacando la microconurbación a dos bandas del Bajo Miño, Chaves-Verín, Vila Real-Peso da Regua-Lamego, Guarda-Covilhã-Castelo Branco, Badajoz-Elvas, y, especialmente, el corredor Faro-Huelva, una vez que ha sido redescubierto por la actividad inmobiliaria y la atracción turística), lo que puede facilitar una estrategia conjunta de complementariedades y sinergias y donde su mejor accesibilidad y la eliminación de las barreras fronterizas posibilitará nuevas oportunidades de desarrollo (L. LÓPEZ TRIGAL, «Le Portugal en Espagne: migrations et société» y R. LOIS GONZÁLEZ, «Galice-Portugal: des relations transnationales privilégiées dans la 
Péninsule Ibérique», in: Sud-Ouest Européen, 18, 2004, pp. 23-30 y 31-40).

\section{A MODO DE CONCLUSIÓN}

Una red emergente de nuevo signo se desarrolla en los últimos años a partir de la animación y las buenas prácticas de las relaciones transfronterizas, de las numerosas inversiones económicas y financieras, de los cada vez mayores desplazamientos turísticos y laborales en las dos direcciones. El modelo de transacciones fronterizas avanza ahora con mayor fuerza en las interrelaciones entre las ciudades de Galicia y Norte de Portugal ('Eixo Atlántico' del Noroeste peninsular), las ciudades y villas ribereñas del Duero/Douro, las universidades de Salamanca y Coimbra, las ciudades del eje Aveiro-Irún, las ciudades y villas rayanas de Extremadura con las de Beira y Alentejo, o las del Algarve y Andalucía occidental. Ciudades y regiones, asociaciones empresariales e instituciones de un lado y otro van tejiendo nuevas relaciones y caminos de cooperación y acción, apoyados en los acuerdos internacionales y las políticas de vecindad y de cooperación e integración europea.

Pero, es preciso advertir que, todo ello está en dependencia con las oportunidades de inserción de las ciudades y su capacidad de estructurar el sistema urbano peninsular y no solo de su mitad occidental, tal como aparece recientemente en las nuevas ligazones aéreas entre Barcelona o Madrid con Porto, entre Bilbao o Valencia con Lisboa, o las más secundarias entre Funchal con Las Palmas o Santa Cruz de Tenerife, pues el espacio de concertación y de cooperación aspira a soldar ambos sistemas nacionales, cada vez más funcionando en un sistema ibérico organizado a partir de las ciudades grandes, en especial Madrid, y las ciudades medias en su papel de protagonizar la intermediación con el territorio y asegurar, de paso, la inserción en las dinámicas de la economía internacional. Por añadidura, este incremento de las relaciones hispanoportuguesas debería posibilitar la dinamización de los pequeños centros urbanos y territorios situados en los espacios fronterizos de la amplia faja rayana, la mayor de vacíos rurales y áreas de pérdida del Suroeste europeo.

Llego al final de mi intervención. Espero que lo dicho haya facilitado el interés de todos los presentes, en especial de nuestro homenajeado el profesor Jorge Gaspar, a quien deseo los mayores augurios desde la representación que me corresponde en este acto por parte de mis colegas españoles. 